\title{
Pyloric stenosis in adults: A clinical and radiological study of 100 consecutive patients
}

\author{
LOUIS KREEL ${ }^{1}$ AND HAROLD ELLIS \\ From the Departments of Radiology and Surgery, Westminster Hospital, \\ London
}

EDITORIAL SYNOPSIS The causes of obstruction in 100 cases diagnosed clinically as 'pyloric stenosis' showed that the majority of cases were duodenal ulcer stenosis, with prepyloric carcinoma as second in frequency. In the earlier stages the obstruction is overcome by hyperactivity of the gastric muscle but later on muscular failure occurs with gastric atony and retention of food. This is reversible and should be corrected before surgical treatment.

This paper presents a study of a consecutive series of 100 adult patients with pyloric stenosis, confirmed in every case at operation, in order to illustrate the clinical and radiological features of this condition, and to discuss the dynamic as well as the purely anatomical aspects of the lesion.

From the pathological point of view the commonly used term 'pyloric stenosis' in the adult patient is inaccurate, since the obstruction is rarely at the pylorus itself but is situated either immediately proximal to the sphincter, where the diagnosis of carcinoma is most likely, or more distally, in the duodenal bulb, where almost invariably the cause is a duodenal ulcer. A more correct designation would be 'gastric outlet obstruction'. However, the term pyloric stenosis is so well established that it is unlikely to be displaced, and we are certainly not suggesting gastric outlet obstruction as a substitute.

The criteria accepted for inclusion of patients in this series were as follows:

1 Clinically, one or more of the following features: projectile vomiting (particularly if food was noticed which had been eaten the previous day), the presence of a gastric splash heard four hours or more after the last meal, visible gastric peristalsis, or a gastric residue of $500 \mathrm{ml}$. or more.

2 Radiologically, all patients had definite evidence of gastric outlet obstruction, which will be discussed later.

3 In all cases the diagnosis of organic 'pyloric' narrowing was confirmed at operation.

The causes of the pyloric stenosis in this series were as follows (Table I). Fifty-six patients had

'Present address: Department of Radiology, The Royal Free Hospital, Gray's Inn Road, London, W.C.1. duodenal ulcer, 36 had carcinoma involving the pyloric antrum, four had benign pyloric ulcers, and two had adult hypertrophy. In addition one patient had Hodgkin's disease involving the gastric outlet and another had an ectopic pancreatic nodule at the pylorus.

TABLE I

100 CONSECUTIVE PYLORIC OBSTRUCTIONS

\begin{tabular}{lc} 
Cause of Obstruction & No. of Cases \\
\hline Duodenal ulcer & 56 \\
Carcinoma & 36 \\
Pyloric ulcer & 4 \\
Adult hypertrophy & 2 \\
Hodgkin's disease & 1 \\
Ectopic pancreas & 1 \\
Total & 100
\end{tabular}

\section{CLINICAL FEATURES}

A comparison of the 56 patients with duodenal ulceration and the 36 with carcinoma of the antral region is of interest (Table II). There were no significant differences in average age between the benign and malignant group (56 and 60 years respectively) nor in the age range or sex distribution (68\% and $59 \%$ for males respectively).

\begin{tabular}{lll} 
& $\begin{array}{c}\text { TABLE II } \\
\text { Duodenal Ulcer }\end{array}$ & Carcinoma \\
\hline Male & $38(68 \%)$ & $21(58 \%)$ \\
Female & 18 & 15 \\
Average age (yr.) & 56 & 60
\end{tabular}

PAIN The length of history of indigestion and epigastric pain, not unnaturally, tended to be 
considerably longer in the patients with benign ulcers (Table III). It is interesting, however, that five patients with duodenal ulceration had a history of pain for less than one year. One of these patients with a history of only two months had had a previous thoraco-lumbar sympathectomy for hypertension and may well have had a consequent gastric denervation. Of the remaining duodenal ulcer patients, 11 had a history of one to four years, 12 of five to nine years, and 27 had a history of from 10 to 20 or more years; in one patient the length of history was not recorded.

In contrast, 11 of the 36 patients with carcinoma had experienced no pain at any time, in 20 the history was under one year, in three the history was up to two years, one was of three years' duration, and in one patient there had been mild dyspepsia for the preceding eight years.

TABLE III

\begin{tabular}{lcc} 
& LENGTH OF HISTORY OF PAIN \\
& Duodenal Ulcer & Carcinoma \\
\hline Under 1 year & 5 & 20 \\
1-4 years & 11 & 4 \\
$5-9$ years & 12 & 1 \\
$10-19$ years & 23 & - \\
$20+$ & 4 & - \\
$?$ & 1 & -11 \\
No pain & - & 36 \\
Totals & 56 &
\end{tabular}

vomiting Vomiting was the commonest presenting symptom in both groups. In the peptic ulcer patients it occurred in 49 of the 56 cases $(87.5 \%)$ and was typical projectile vomiting of large amounts in 39 of these. In addition, 24 of the patients noted stale food in the vomitus. In the carcinoma group 31 of the 38 patients $(86 \%)$ had been vomiting; in 23 of them it was typical of stenosis and in nine stale food had been noted in addition.

OTHER SYMPTOMS Weight loss was a prominent feature in both groups, occurring in 44 of the duodenal ulcer patients $(78 \%$ ) and in 34 patients with cancer $(94 \%)$.

Recent constipation was recorded in 14 of the duodenal ulcer and in $\mathbf{1 2}$ of the carcinoma group.

Recent diarrhoea was recorded in three of the ulcer and in three of the cancer series.

Gastrointestinal bleeding had occurred recently in five patients with duodenal ulcer and at some time in the past in eight further cases.

In the carcinoma group eight patients had small recent haemorrhages; one indeed presented with melaena and another with anaemia.

Three of the ulcer series had perforations repaired in the past but there was no case of associated perforation in the cancer group.

\section{EXAMINATION}

On clinical examination it is obvious that the 'typical ulcer facies' of the old text books applied to many of the duodenal ulcer patients with stenosis. Thirty-three patients were thin $(59 \%)$ and only one of the whole group was described as obese. Abdominal examination revealed a splash in 36 patients of the ulcer group ( $64 \%$ ), in nine of whom, in addition, visible peristalsis was seen and in four of whom the gastric distension was great enough to produce a large, palpable mass. In contrast, only five of the patients with carcinoma had a splash $(14 \%)$ and only one had visible peristalsis. However, 19 of the patients with carcinoma had a palpable mass in the pyloric region (Table IV).

\begin{tabular}{lcc} 
& TABLE IV & \\
& Duodenal Ulcer & Carcinoma \\
\hline Mass & - & $19(53 \%)$ \\
Splash & $36(64 \%)$ & $5(14 \%)$ \\
Stomach visible & 9 & 1 \\
Stomach palpable & 4 & - \\
& &
\end{tabular}

Biochemical disturbances did not occur in patients who had never vomited or in whom the vomiting was not profuse or of almost daily occurrence. In 44 patients in this series, vomiting had been copious and frequent before admission; in 20 of these the blood biochemistry was completely normal, and in a further 11 there was only a raised blood urea above $50 \mathrm{mg} . \%$ as the sole biochemical abnormality, in six being above $100 \mathrm{mg} . \%$ and in one of these reaching $243 \mathrm{mg} . \%$. All were rapidly corrected after rehydration. In the remaining 13 patients there were more widespread biochemical alterations including, in nine of these, a raised blood urea. In these 13 cases there were one or more of the following changes: sodium less than $135 \mathrm{mEq} . \%$, potassium less than $3.5 \mathrm{mEq} . \%$, chloride less than $95 \mathrm{mEq} . \%$ or bicarbonate above $30 \mathrm{mEq} . \%$ (Table V).

It is interesting that only five of the 24 patients with biochemical disturbance were admitted in a clinically obvious state of electrolytic imbalanceill, dehydrated, weak and, in one case, with obvious tetany.

Anaemia (haemoglobin of less than $80 \%$ ) was present in 11 of the 56 patients with duodenal ulceration and in seven of the 36 with carcinoma: in one of the latter it was the presenting feature.

\section{ASSOCIATED BENIGN GASTRIC ULCER}

Four of the patients with duodenal ulceration had an associated benign gastric ulcer confirmed at 
TABLE V

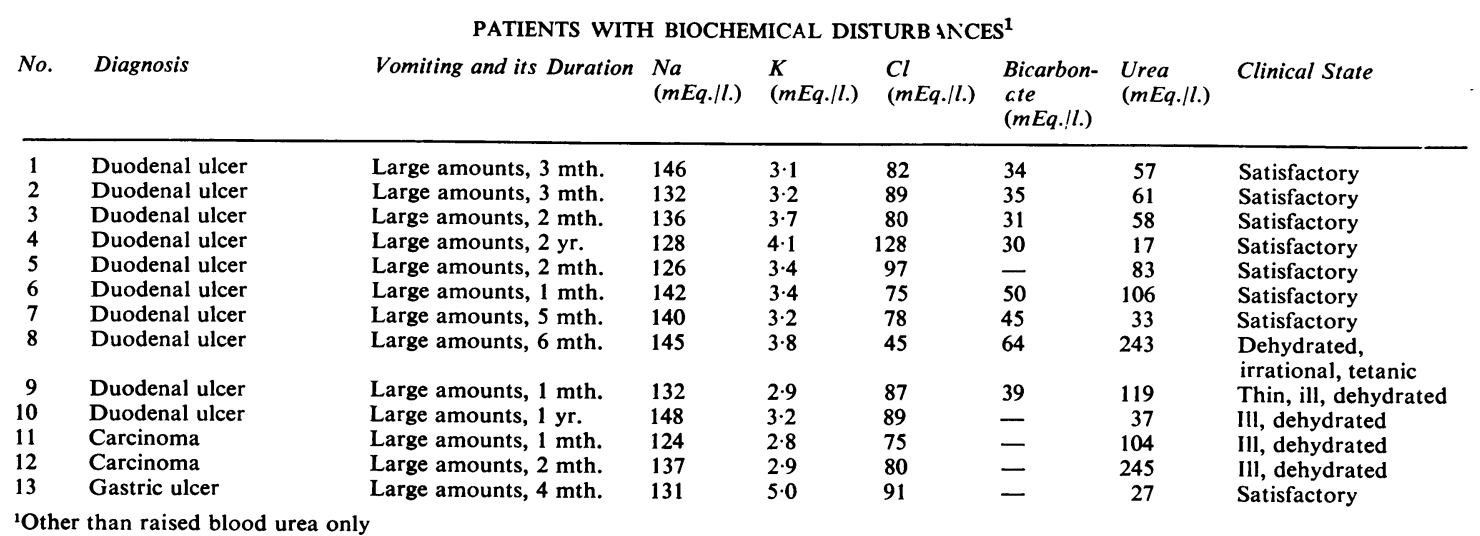

operation. Three of these were women. Two more patients, also women, had radiological evidence of a gastric ulcer, although as these patients had drainage procedures and not partial gastrectomy, histological confirmation was not obtained. Apart from this quite marked sex bias for gastric ulcer associated with stenosis due to duodenal ulcer, analysis revealed no relevant differences from the remaining patients with benign pyloric obstruction.

\section{RADIOLOGICAL AND BARIUM MEAL EXAMINATION}

The radiological signs of pyloric stenosis can be divided into those indicating the presence of obstruction at the gastric outlet and those indicating its site and possible nature. However, very uncommonly, the first radiological abnormality to be detected is the presence of lymphangitis carcinomatosa on the chest film (one case).

Occasionally there is evidence of gastric stasis on the plain film of the abdomen in the form of a large gastric shadow with retained food particles producing patchy translucencies (two cases). In these cases an erect film will show a large, high gastric fluid level. Retention of oral cholecystographic contrast from the previous evening with a 'non-functioning' gall bladder was the first radiological evidence of obstruction at the gastric outlet in two cases (Fig. 1). Subsequent barium meal examination revealed a deformed, contracted duodenal cap as the cause of the obstruction.

SIGNS OF OBSTRUCTION AT THE GASTRIC OUTLET There are several signs of obstruction at the gastric outlet visible radiologically.

Presence of excessive fasting gastric juice This is immediately apparent on the first mouthful of barium reaching the stomach. Instead of the bolus

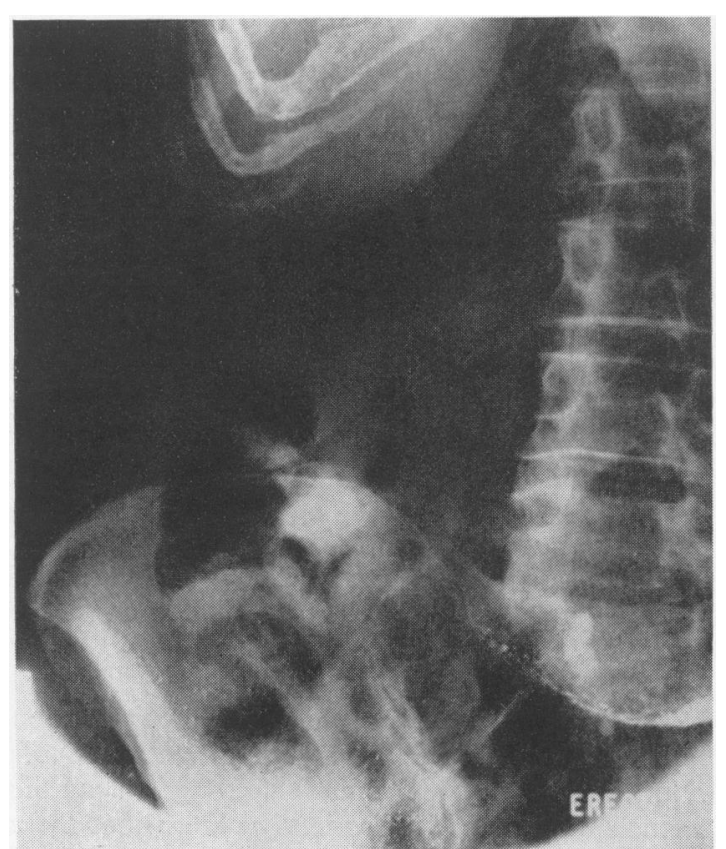

FIG. 1. Oral cholecystographic contrast medium retained in the stomach 14 hours after ingestion of the tablets. In two cases in this series this was the first radiological sign of gastric outlet obstruction.

descending along the lesser curve and parting the opposed gastric walls, the blobs of barium sink through a layer of fluid and come to rest at the bottom of the greater curve giving a saucer-like appearance. Thus the film in the erect position shows three media: air, resting juice, and barium, with two intermediate fluid levels (Fig. 2).

Excessive peristalsis While the stomach is in the 'compensated' phase, it shows two to three giant 


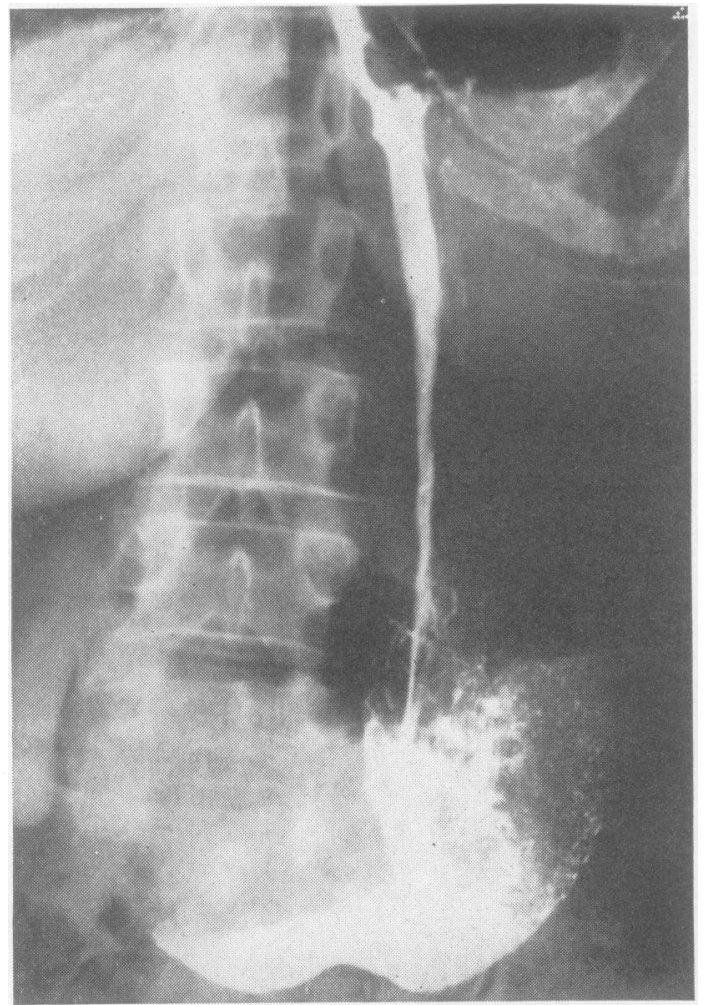

FIG. 2. Barium entering the stomach in the presence of excessive gastric juice showing three media-barium, gastric juice, and air-and the two intervening fluid levels.

peristaltic waves which indent both the greater and lesser curves simultaneously. These giant waves serve to distinguish the large active stomach of pyloric stenosis from the obstructed atonic organ (Fig. 3).

However, in the phase of failing compensation these peristaltic waves peter out after a variable period (one to three hours) leaving an atonic stomach. It thus not infrequently occurs that there is good emptying through the area of stenosis initially, and yet a significant barium residue is present in the stomach at four to six hours. The presence of giant peristaltic waves is thus an indication that a delayed film must be taken to assess the barium residue.

Large atonic stomach The large atonic stomach is found in both gastric outlet obstruction and in functional stasis. However, in the latter condition barium flows freely through the pylorus if the patient is correctly postured, i.e., prone with the left side raised. The large stomach is more common with benign than with malignant lesions as has been noted under clinical features above.
Delay in emptying A 24-hour barium residue is usually diagnostic but this is seldom necessary. A four to six-hour film, using barium sulphate as the contrast medium, normally shows less than $20 \%$ retention whereas in pyloric stenosis there is more than $50 \%$ retention. It is, however, important to exclude pylorospasm or obstructive lesions in the proximal small bowel. Pylorospasm can be distinguished by administering probanthine intravenously, after which the stomach will empty normally.

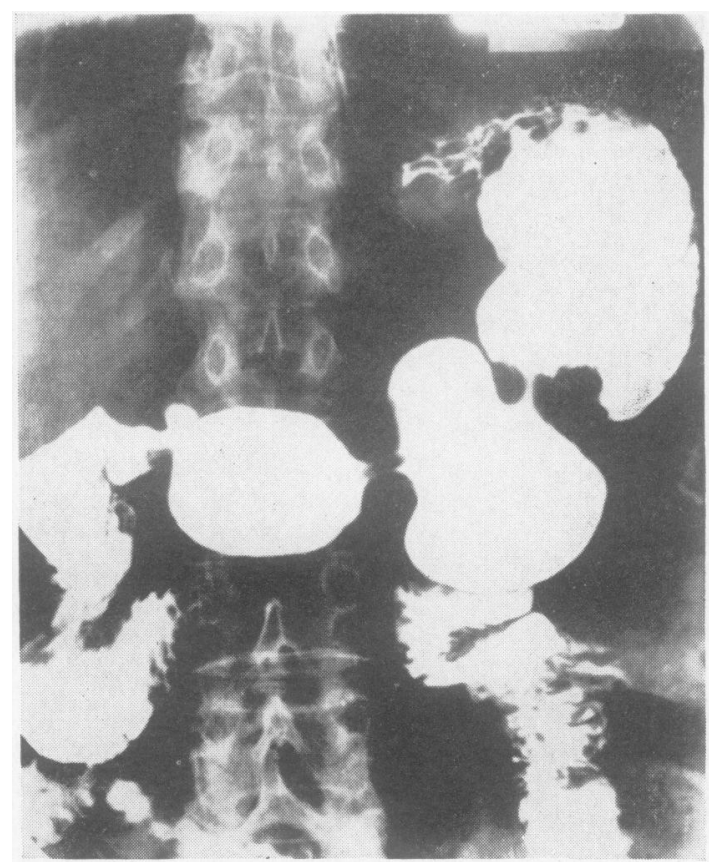

FIG. 3. Giant peristaltic waves in the 'compensated' phase of pyloric obstruction which, in this case, is due to duodenal scarring with an associated penetrating ulcer. At this stage no barium was present at six hours. Four months later this patient was re-admitted in the 'decompensated' phase with a large atonic stomach.

Obstructive lesions in the second and third parts of the duodenum or in the proximal part of the jejunum may show all the radiological and clinical features of pyloric stenosis.

Abnormal mobility of the pylorus Where the site of obstruction is beyond the pylorus, as in cicatrization of the duodenal cap, the pyloric canal shows abnormal mobility. The pyloric canal may dilate intermittently, up to $2.5 \mathrm{~cm}$. in width, and thus appear to be part of the gastric antrum, but further observation will show it to contract down to its usual size. This abnormal dilatation (Fig. 4) of the pyloric canal, we have found, is a reliable sign of duodenal bulb obstruction and could be demon- 


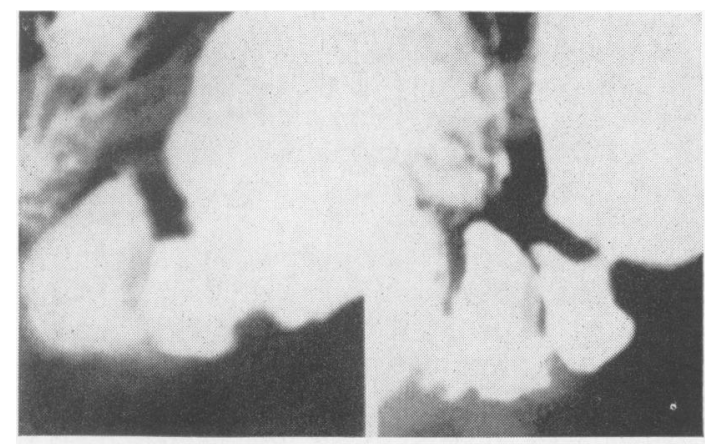

ing mural lesion. The margins of the tapered antrum tend to be irregular and the wall is rigid. It can only be moved in toto, if at all, and does not indent on palpation. Moreover there is a lack of peristalsis, so that films taken at various intervals can be accurately superimposed.

The superior margin of the rigid, rounded antrum (two cases) is often irregular and again accurate superimposition of successive films is possible (Fig. 5). A filling defect within the antrum, due to the carcinoma, may be detected on gentle pressure.

An antral mass with destruction of the mucosal pattern was found in 18 cases. A large irregular

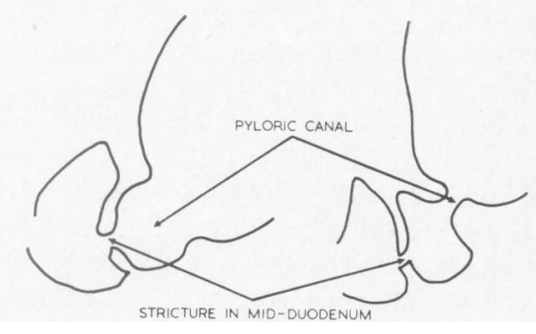

FIG. 4. Two adjacent spot films from a serial showing abnormal 'dilatability' of the pylorus proximal to a stricture of the mid-duodenal bulb.

strated in almost all patients (50 of 56 cases). This appearance may also occur, but to lesser extent, in obstructive lesions elsewhere in the duodenum, e.g., arterio-mesenteric occlusion, or carcinoma of the duodenum.

SIGNS INDICATING THE SITE AND NATURE OF OBSTRUCTION The cause of obstruction at the gastric antrum is almost always malignancy although obstruction may rarely be caused by cicatrization from peptic ulcer, corrosives, or chronic inflammatory lesions such as tuberculosis and syphilis. Occasionally the malignant process arises outside the stomach as in pancreatic carcinoma or in Hodgkin's disease of the abdominal lymph nodes.

The radiological signs of malignancy are the rigidity of the antral wall, its lack of peristalsis, and its irregular contour. The rigidity is indicated by its lack of movement on positioning and on palpation. Successive films can be superimposed with accuracy, accounting for the 'rigid' quality seen on films. The irregular contour often affects the superior margin of the antrum and may be associated with the absence of the mucosal pattern.

The tapered antrum (14 cases) may be produced by a mass surrounding the antrum or by an infiltrat-

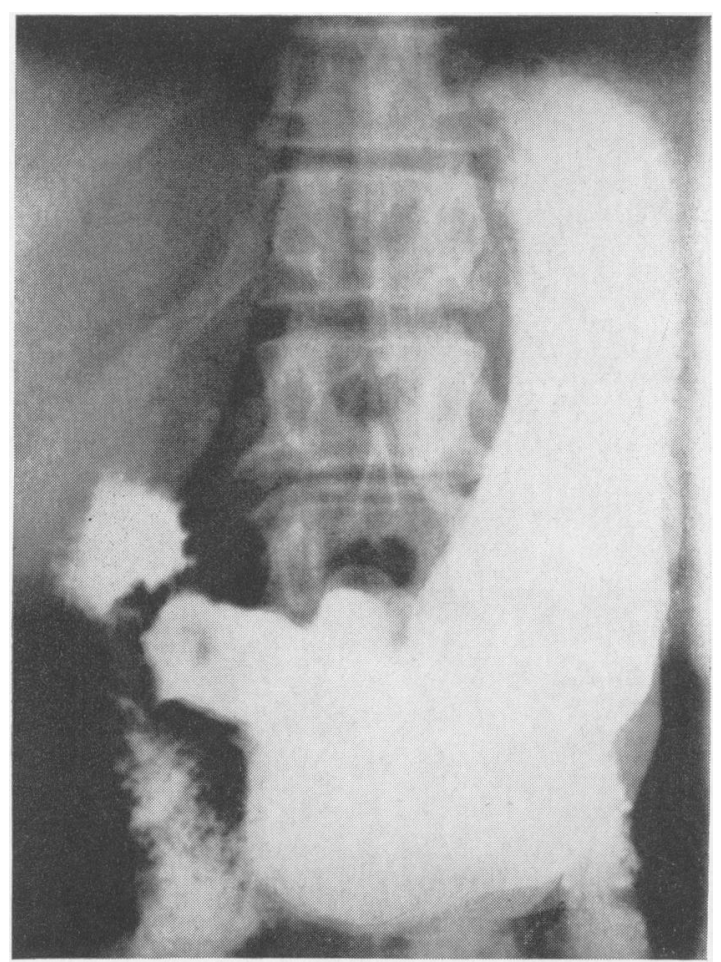

FIG. 5. Three superimposed exposures on one film to demonstrate the rigidity of the gastric antrum compared with the mobility elsewhere. The filling defect of the carcinoma can be seen in the antrum.

filling defect, usually arising from the greater curve, may cause obstruction at the gastric outlet. There is usually little doubt about this appearance being malignant, but just occasionally the granulation tissue surrounding a penetrating posterior wall gastric ulcer produces similar appearances, including the absence of a mucosal pattern (Fig. 6). 


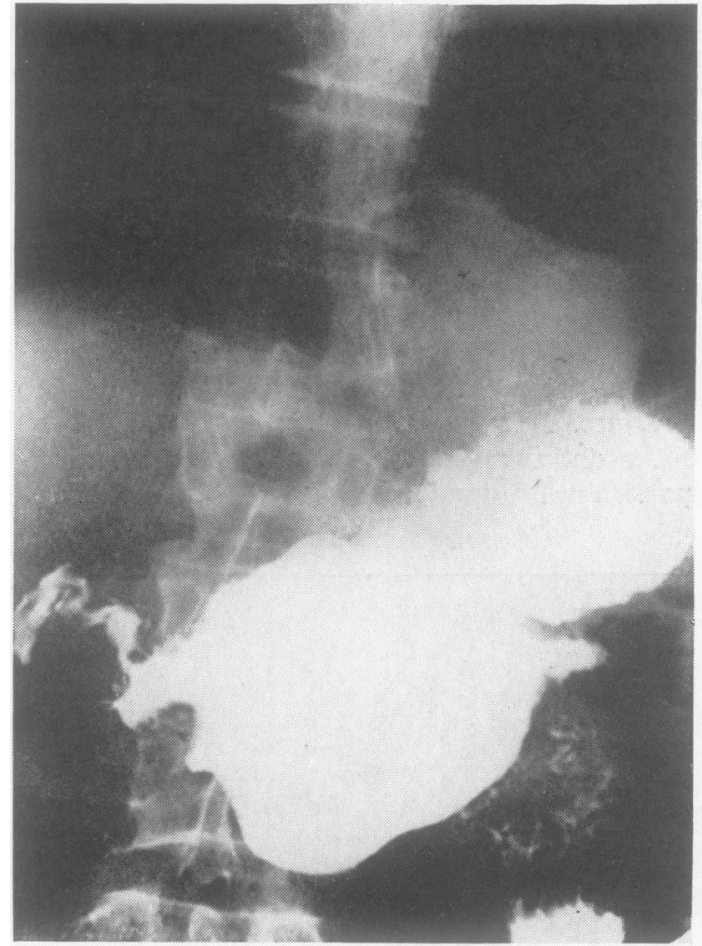

FIG. 6. A benign posterior wall gastric ulcer with excessive granulation tissue mimicking a carcinoma of the antrum by producing a large filling defect on the greater curve with associated obstruction.

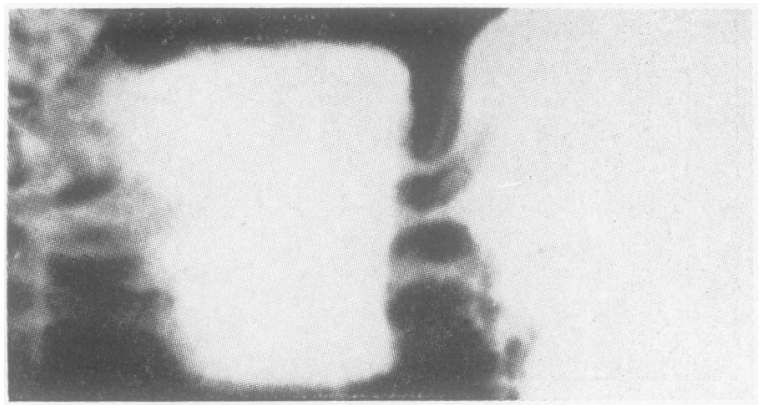

FIG. 7a. A radiograph of the closed pylorus, the mucosal furrows in the pyloric ring trapping barium to give a picture of white lines.

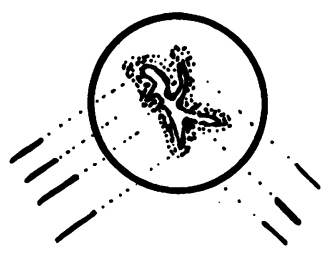

FIG. 7b. The slit lumen of the pylorus is asymmetrical. The distance between the barium streaks will depend on the direction of the rays and does not correspond to the diameter of the canal.
A carcinomatous ulcer may be associated with obstruction of the gastric outlet.

Recurrence around a gastric stump following gastrectomy produces appearances similar to the tapered antrum above but now at the gastric stump outlet. (One case, which is not included in this series, was seen.)

Lesions occurring at the pyloric canal may produce obstruction, but this is in fact, the least common site of obstruction of the gastric outlet.

Obstructive symptoms and signs may appear in the presence of active ulceration, when a persistent barium fleck or ulcer niche will be visible. More commonly, the obstruction occurs following cicatrization. The canal is then narrowed and elongated. In this respect it is important to note that the diameter of the canal does not correspond to the width between the barium streaks in the pyloric canal (Fig. 7) as was shown by Williams (1962). We include four cases in this series.

Adult hypertrophic pyloric stenosis (two cases) can be recognized by the bulbous intrusion into the base of the cap, the spikes along the canal, and the length of the narrowed segment. Occasionally the impression on the base of the cap is so marked as to produce a circular filling defect in the duodenal bulb (Fig. 8).

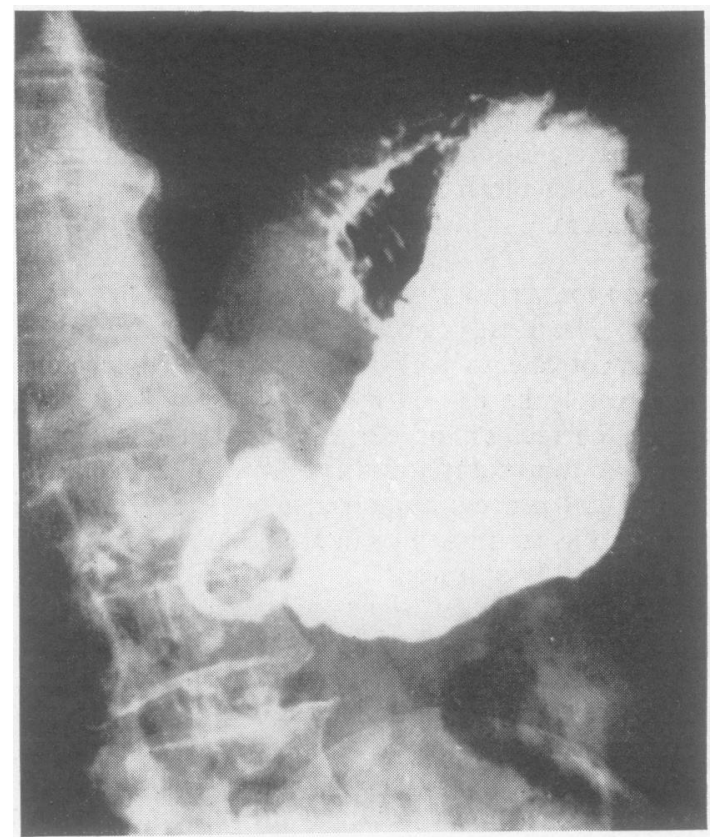

FIG. 8. Film of adult hypertrophic pyloric stenosis in which the impression on the base of cap by the hypertrophied pylorus was so marked as to produce a circular filling defect in the duodenal bulb. 
Although ectopic pancreatic tissue may occur in the antrum and duodenum it seldom causes obstruction. However, if it is situated at the pylorus this may occur. The clue to its presence lies in the demonstration of a thin streak of barium communicating with the pyloric lumen. This represents barium in the duct of the ectopic pancreas (Fig. 9). One case is included.

Scarring of the duodenal cap may take one of several forms, but our cases have fallen into the following groups:

1 Eccentric scarring of the superior or lesser curve wall with the lower fornix forming the prestenotic diverticulum of Akerlund. This was the most common appearance in our series (36 cases).

2 Central scarring producing a dumb-bell appearance (three cases).

3 Central scarring forming an irergular starshaped configuration (nine cases).

4 Pyloric stenosis caused by peptic ulceration is often associated with active ulceration. In the duodenum the ulcer is often of the penetrating type producing the appearance of an 'irregular diverticulum', which tends to remain constant in size and

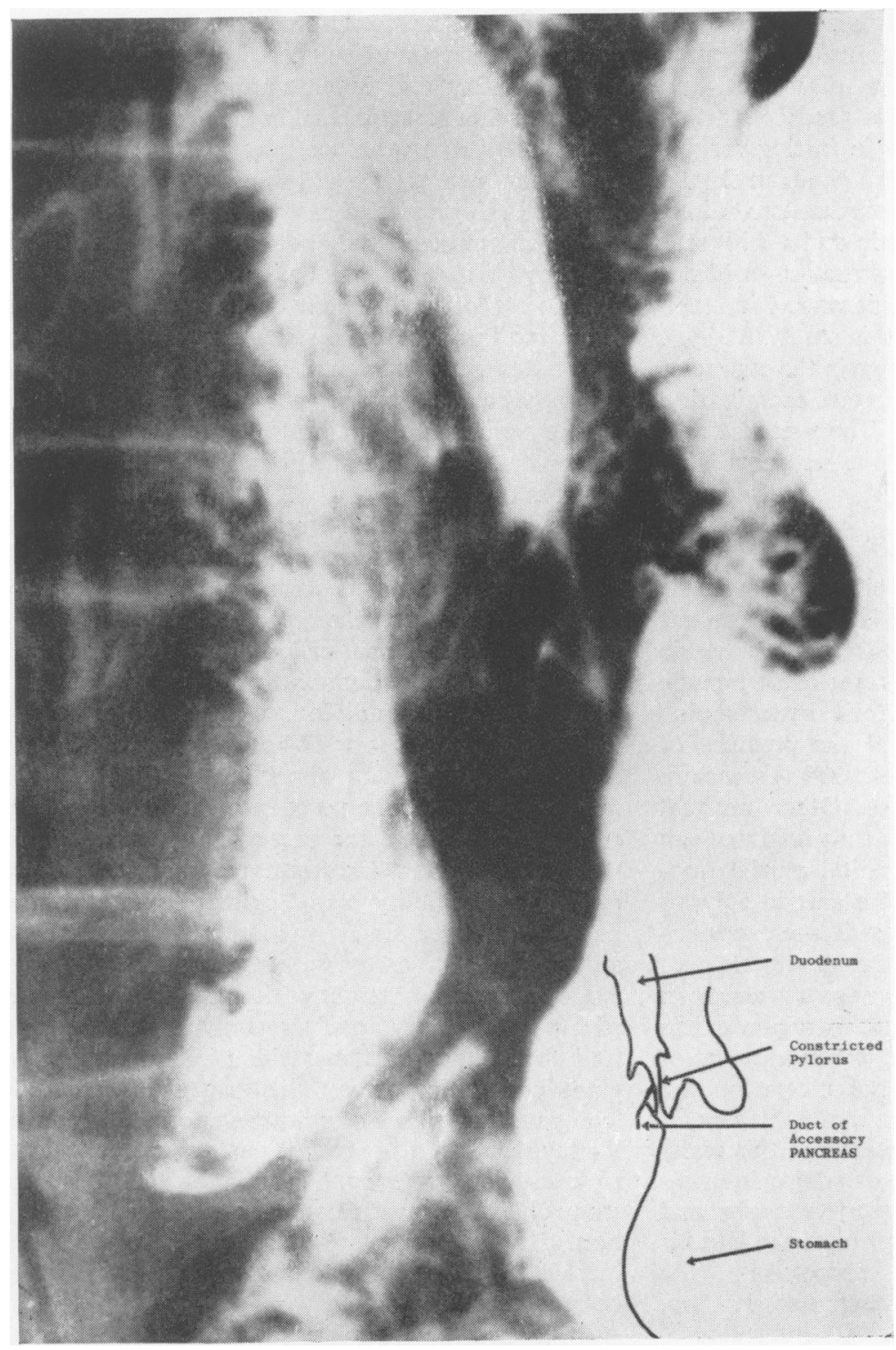

FIG. 9. Obstruction caused by heterotopic pancreatic tissue encircling the pyloric canal. Note the barium streak outlining the ectopic pancreatic duct. 
position and most frequently occurs on the posterosuperior aspect of the duodenum. At operation it is frequently associated with adherence of the duodenum to the liver or the pancreas by a mass of granulation tissue.

5 As previously mentioned an associated gastric ulcer was present in six of 56 patients with duodenal ulceration.

\section{DISCUSSION}

AWARENESS OF EARLY DIAGNOSIS The diagnosis of established pyloric stenosis is not a difficult one to make; there are the typical features of projectile vomiting, the presence in the vomitus of food eaten one or more days previously, loss of weight, and loss of appetite. On examination a gastric splash is present and there is a considerable fasting residue on gastric aspiration. However, the clinician should be aware of the fact that the chronic duodenal ulcer, as it gradually constricts the gastric outlet with scar tissue, may produce gross obstruction without vomiting ever having occurred; this was so in seven patients with duodenal ulcer in this study $(22.5 \%)$. It is easy enough to miss this change in the character of a patient's pathology unless a careful examination is carried out at each attendance. The presence of a gastric splash several hours after the last meal is an important sign and may occur without the presence of any warning symptoms.

PATHOLOGY AND DIFFERENTIAL DIAGNOSIS As is seen in Table I, duodenal ulcer is the commonest cause of pyloric obstruction, followed by pyloric carcinoma. Other causes are all rare; these include pyloric gastric ulcer, benign pyloric hypertrophy, heterotopic pancreatic tissue, and the pressure of enlarged adjacent lymph nodes. All these are represented in the present series. Other unusual causes are scarring due to a chronically inflamed gall bladder, annular pancreas, congenital partial duodenal diaphragm, the prolapse of a gastric polypus through the pylorus, chemical strictures, eosinophilic granuloma, and extrinsic fibrous adhesions.

The differential diagnosis between the two common conditions of duodenal ulcer and carcinoma can often be made clinically with some degree of accuracy. In the case of duodenal ulceration the process is usually a long one and the history of dyspepsia in two-thirds of the patients in this series was five or more years. The long-standing stenosis enables the stomach to dilate and hypertrophy and this was summarized in the aphorism of the late Sir James Walton, who described successively 'the stomach one can hear, the stomach one can see, and the stomach one can feel'. At first there is sufficient residue in the stomach for a gastric splash to be elicited; as the stomach hypertrophies visible gastric peristalsis may be seen; finally the thickened stomach, full of inspissated contents, becomes palpable.

In contrast, the length of history in gastric carcinoma is usually short and indeed in about onethird of cases there is no story of abdominal pain at all. The short period of obstruction does not allow gastric hypertrophy to take place and even the presence of a gastric splash in these cases is unusual. On the other hand careful palpation will often reveal the presence of an abdominal mass in the region of the pylorus. The detection of such a mass is not entirely diagnostic of tumour. Rarely one finds that the thickening and inflammation around the duodenum and pancreatic head in the presence of a large active ulcer may produce a very similar mass; we have seen two such examples, although none was present in this series of cases.

To summarize, the patient with the typical features of a duodenal ulcer which progresses to pyloric stenosis will clinically have a long history, show marked loss of weight, and a gastric splash will be present. The patient with carcinoma will typically have a relatively short history, may in fact have experienced no pain at all, and will frequently demonstrate an abdominal mass without evidence of gross dilatation.

The pre-operative diagnosis of the rarer conditions producing stenosis is usually only possible radiologically and even then may not be accurate. The surgeon must therefore be aware of these unusual causes when called upon to perform a laparotomy on a patient with pyloric obstruction.

There are few radiologists who routinely take four to six-hour films or 24-hour films after a barium meal. If patients with abnormal gastric stasis are not to be missed, one must be aware of those signs which may indicate the presence of obstruction of the gastric outlet. Excessive resting gastric juice, excessive dilatability of the pyloric canal and giant peristaltic waves, singly or in combination, call for a delayed film to assess barium retention.

However, an obstructive lesion may be present with normal emptying of the stomach and a normal four to six-hour barium residue in the presence of giant peristaltic waves. This has been called the 'compensated' phase of pyloric stenosis (Shanks and Kerley, 1958). This excessive gastric peristalsis leads to muscular hypertrophy and thickening of the stomach wall, as was frequently confirmed at operation. This hypertrophy not infrequently extends to include the lower oesophagus. This may on occasion be recognized radiologically.

In patients with benign lesions such as duodenal 
ulcer scarring and adult hypertrophic pyloric stenosis, the exit channel may be extremely narrow and yet there may be little or no gastric retention.

Gastric 'failure' leading to the onset of marked retention of gastric juice, and food residue is thus not entirely dependent on the degree of obstruction. The high incidence of associated penetrating ulcers in the duodenal ulcer cases suggests that reactivation of the ulcer may be a precipitating factor.

Gastric muscle failure may occur as a consequence of this overactivity, the giant peristaltic waves petering out before the stomach is entirely empty. The gastric residue then builds up, and is not relieved even by a night's rest. The fasting 'gastric' juice under these circumstances may indeed be largely due to swallowed saliva. We have, unfortunately, not been able to devise a method for separating swallowed saliva from gastric secretion and thus to tell whether the fasting 'gastric' juice originates mainly in the mouth or in the stomach.

It may indeed be that the decompensated phase of pyloric stenosis, with gastric atony and marked gastric retention, is caused by an inherent muscular failure, dependent on intracellular potassium or magnesium transference. In this respect the gastric muscle failure may be similar to cardiac muscle decompensation in stenotic valvular heart disease.

The ultimate cause of the gastric muscle failure or atony in pyloric stenosis is obscure, but it is clear that most patients are diagnosed long before this late stage in the disease is reached.

THE IMPORTANCE OF DIAGNOSIS The diagnosis of pyloric stenosis is an absolute indication that surgical exploration of the abdomen is required. It is important that the clinician and radiologist establish the diagnosis pre-operatively. Metabolic disturbances may be present which will require careful correction before operation, and moreover the dilated stomach with its secondary gastritis is a dangerous organ upon which to operate. The value of pre-operative diagnosis is that this enables the surgeon to prepare his patient carefully by gastric washouts, fluid and electrolyte replacement, and a strict medical ulcer régime. This will result in a considerable improvement both in the general condition of the patient and in the tone of the stomach wall. As the active ulcer heals and the surrounding oedema diminishes, the condition reverts to the compensated phase in which the motor power of the stomach is able to overcome the pyloric obstruction once more.

It may be that enthusiasm on the part of the clinician may result in occasional diagnoses of organic pyloric obstruction being made when in fact this condition does not exist. This may happen, for example, in the presence of a duodenal ulcer with marked pylorospasm without actual organic stenosis or in an atonic stomach with the presence of a gastric ulcer remote from the duodenum. In other cases excessive vomiting with loss of potassium may result in temporary gastric atony which may simulate stenosis. These possibilities are not common; there are usually in any case other indications for surgical intervention and it is safer for the surgeon to err on the side of too frequent rather than too infrequent diagnosis of this condition.

We should like to thank the physicians, surgeons, and radiologists at the Westminster Hospital who allowed us free access to their notes and radiographs.

Our thanks are also due to the Photographic Department of the Royal Free Hospital for preparing the reproductions.

\section{REFERENCES}

Shanks, S. C., and Kerley, P. J. (1958). A text-book of X-ray diagnosis, 3rd ed., vol. 3, p. 128. H. K. Lewis, London.

Williams, I. (1962). Closure of the pylorus. Brit. J. Radiol., 35, 653-670. 